\title{
Finite Element Modeling of a Vibrating Touch Screen Actuated by Piezo Patches for Haptic Feedback
}

\author{
Buket Baylan, Ugur Aridogan, and Cagatay Basdogan \\ College of Engineering, Koc University, Istanbul, 34450, Turkey \\ \{bbaylan, uaridogan, cbasdogan\}@ku. edu.tr
}

\begin{abstract}
The aim of our work is to design a touch screen for displaying vibrotactile haptic feedback to the user via piezo patches attached to its surface. One of the challenges in the design is the selection of appropriate boundary conditions and the piezo configurations (location and orientation) on the screen for achieving optimum performance within the limits of human haptic perception. To investigate the trade-offs in our design, we developed a finite element model of the screen and four piezo actuators attached to its surface in ABAQUS. The model utilizes the well-known Hooke's law between stress and strain extended by piezoelectric coupling. After selecting the appropriate boundary condition for the screen based on the range of vibration frequencies detectable by a human finger, the optimum configuration for the piezo patches is determined by maximizing the vibration amplitude of the screen for a unit micro Coulomb charge applied to each piezo patch. The results of our study suggest that the piezo patches should be placed close to the clamped sides of the screen where the boundary conditions are applied.
\end{abstract}

Keywords: touch screen, vibrotactile haptic feedback, finite element modeling, piezo patch actuators.

\section{Introduction}

The touch screens replace the mechanical buttons on mobile devices, touch pads, tablet PCs and other displays. While the screens available in the market today are sensitive to touch inputs and gestures, they do not enable the user to feel any programmable resistive forces as her/his finger moves on its surface. However, it is desirable to display some of the information through haptic channel in mobile devices, touch pads, tablet PCs and other interactive displays in order to alleviate the perceptual and cognitive load of the user since our visual and auditory channels are already highly overloaded. Moreover, haptic feedback is more personal and intimate than visual and auditory feedback and hence can enrich the user experience and perception of the interaction. We anticipate that the use of haptic feedback as an additional information channel in interactive displays will result in a new interaction paradigm, and enable novel applications in games, entertainment, education, internet-based business, and many more. 
So far, various approaches have been followed to display haptic feedback on touch surfaces. Almost one and a half decade ago, Kaczmarek et al. [1] developed a touch surface for electrocutaneous stimulation of user's finger pad by applying current to it via the electrodes placed on the surface. They reported the difficulty of altering the tactile perception of the user by adjusting the applied current. Later, Kaczmarek et al. [2] developed another touch surface based on electrostatic actuation using a matrix of $7 \times 7$ pin electrodes, which are covered with an insulator layer to prevent direct contact of the finger pad with the electrodes. They conducted a user study and investigated the tactile perception of subjects by displaying four different biphasic waveforms through the touch surface. The results of the study showed that the sensitivity of the subjects to the positive pulses was less than that of the negative or biphasic pulses. Yamamato et al. [3] developed a telepresentation system for tactile exploration of remote surface textures. This system was made of two parts: a tactile sensor on the slave site and a tactile display utilizing an electrostatic actuator on the master site. As the user moves her/his finger on the display, the tactile sensor simultaneously scans the texture surface and the surface roughness recorded by the sensor is displayed to the user through the tactile display by applying two-phase cyclic voltage patterns to the electrodes. They conducted a user study and reported that the subjects correctly matched the textures at the remote site to the local ones with a success rate of $79 \%$. Bau et al. [4] from Disney Research presented TeslaTouch, a touch screen providing haptic feedback to the user based on electrostatic actuation. The device controls the frictional force between the user finger and the screen by modulating the frequency and the amplitude of alternating electrostatic force. The results of the psychophysical studies performed with 10 subjects showed that the average frequency JND varied from $11 \%$ at $400 \mathrm{~Hz}$ to $25 \%$ at $120 \mathrm{~Hz}$ and the average amplitude JND was $1.16 \mathrm{~dB}$ and constant across all frequencies. Maaski and Toshiaki [5] integrated electric motors into a PDA to develop a vibrotactile haptic interface, which they called it "active click". They suggested that active click can improve the usability of touch panels, especially in noisy environments. Poupyrev and Maruyama [6] utilized piezo film actuators to design a PDA with haptic feedback to the user. They argued that further research is required to improve the quality of haptic interactions by developing formal design guidelines. Biet et al. [7] developed a tactile display using an array of piezo actuators attached to the back side of a metal plate. The plate was vibrated at an ultrasonic resonance frequency of $30.5 \mathrm{kHz}$, reaching to peak-to-peak amplitude of $2.3 \mu \mathrm{m}$ and causing a squeeze film to form between the surfaces of the user's finger and the plate. By controlling the thickness of the squeeze film, square gratings were simulated. They conducted a user study with 12 subjects and investigated the slipperiness thresholds of the square gratings for the vibration amplitudes of $0,0.5,1.2$, and 2.3 $\mu \mathrm{m}$. Winfield et al. [8] developed $\mathrm{TPaD}$ by attaching a piezo disk to a glass plate. The plate was actuated at ultrasonic frequencies to modulate the friction coefficient between the finger and the plate surface based on the squeeze film effect. Chubb et al. [9] further extended this idea in ShiverPaD [9] by oscillating the plate in-plane at a frequency of $854 \mathrm{~Hz}$ using a voice coil. Hence, the ShiverPaD is capable of applying and controlling shear force on a finger regardless of its direction of motion. 
Our group designs a touch screen actuated by piezo patches for displaying vibrotactile haptic feedback to the user. One of the challenges in the design is the selection of appropriate boundary conditions and the piezo configurations on the screen for achieving optimum performance within the limits of human haptic perception. To investigate the design trade-offs, we developed a finite element model of the screen and four piezo actuators attached to its surface in ABAQUS. The model utilizes the well-known Hooke's law between stress and strain extended by piezoelectric coupling. After selecting the appropriate boundary condition for the screen based on the range of vibration frequencies that are detectable by a human user, the optimum configuration for the piezo patches is determined by maximizing the vibration amplitude of the screen while minimizing the power consumption of the piezo actuators.

\section{Our Approach}

To demonstrate our idea, we attach an array of thin-film piezo actuators at the back surface of a glass plate where computer-generated images are projected onto it through an LCD display (Figure 1). These piezo films are actuated by a signal generator and an amplifier to generate vibrations on the front surface of the plate with varying amplitudes and frequencies. The position of the user finger on the glass plate is sensed by an IR frame.

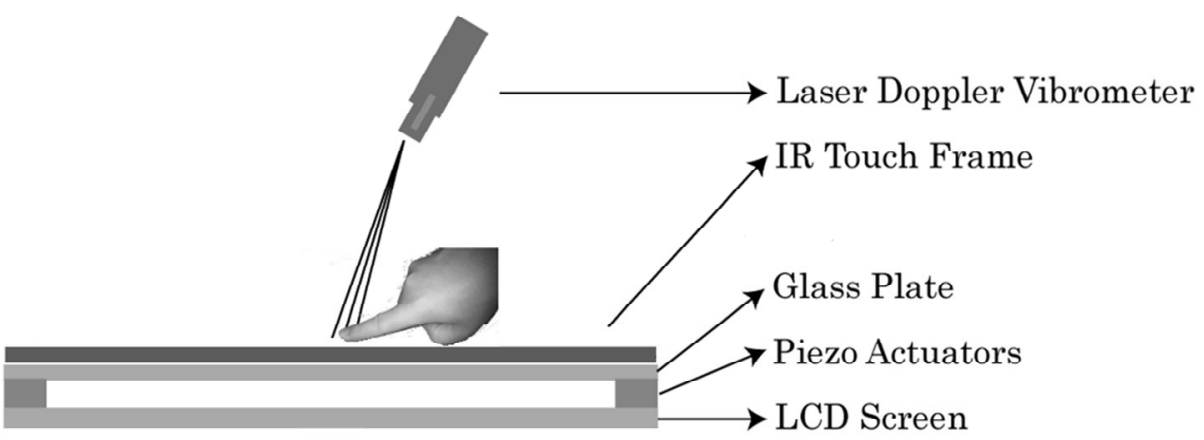

Fig. 1. The proposed vibro-tactile display: The user feels the vibrations generated by an array of piezo actuators glued to the back surface of a glass plate. The magnitude, frequency, and direction of the vibrations are tailored to induce application-specific tactile sensations on the user.

In order to construct the system shown in Figure 1, we first decided on the type of thin-film piezo actuators and then purchased them from the manufacturer (PI Dura-act $\mathrm{P}-876 . S P 1)$. In addition to being small and thin $(16 \times 13 \times 0.5 \mathrm{~mm})$, this type of piezoelectric patches are light-weighted (0.3 gram), hence the additional weight due to coupling with the glass plate is negligible compared to the own weight of the plate. Besides these advantages, the insulation layer enables it to be attached to the glass plate easily. We glued one of the piezo patches on the glass plate $(230 \times 180 \times 3 \mathrm{~mm})$ 
and conducted some initial experiments by applying alternating voltage to the patch through a signal generator and an amplifier to vibrate the plate (Figure 2). We observed that the vibration amplitudes generated by a single patch are not sufficient to be sensed by a human finger.



Fig. 2. Our experimental set-up

Hence, we decided to investigate the optimum number and placement of piezo patches on the glass plate. Since the piezo patches cannot be easily detached from the glass plate once they are glued to its surface, it is more convenient to make this analysis in simulation environment using ABAQUS finite element package. For this purpose, we developed the models of the glass plate, the piezo patch actuators, and the interactions between them in ABAQUS and then investigated the design trade-offs based on the amplitude and frequency of the vibrations of the glass plate and the power consumption of the patches.

For the glass plate and the piezoelectric actuator, element types C3D20 and $\mathrm{C} 3 \mathrm{D} 20 \mathrm{E}$ are used respectively. The material properties of the glass plate are taken from the literature. The material and electrical properties of the piezo patches are provided by the manufacturer. Our initial design consists of four piezoelectric actuator patches attached to a glass plate in 8 different configurations of the patches (Figure 3). By running simulations in ABAQUS, we first investigated the effect of 3 different boundary conditions on the resonance frequency of the plate and then the effect of piezo locations and orientations on the amplitude of the vibrations of the plate per unit charge applied to the piezo patches.

\section{$3 \quad$ Modeling}

The electro-elastic response of the glass plate is governed by the following finite element equations [10] [11]: 


$$
\begin{aligned}
& M \ddot{U}+K_{u u} U+K_{u \Phi} \Phi=F \\
& K_{\Phi u} U+K_{\Phi \Phi} \Phi=G
\end{aligned}
$$

where, $M$ is the mass matrix, $K_{u u}$ is the stiffness matrix, $K_{u \Phi}$ and $K_{\Phi u}$ are the piezoelectric coupling matrices, $K_{\Phi \Phi}$ is the capacitance matrix, $U$ is the ment, $\Phi$ is the electrical potential, $F$ is the externally applied force and $G$ is the applied charge. In our case only charge is applied and the externally applied force is zero. Substituting zero for F into Eq. 1,

$$
\begin{aligned}
& M \ddot{U}+K_{u u} U+K_{u \Phi} \Phi=0 \\
& M \ddot{U}+K_{u u} U=-K_{u \Phi} \Phi
\end{aligned}
$$

and solving for $\Phi$ using Eq. 2, we obtain

$$
\Phi=K_{\Phi \Phi}{ }^{-1}\left[G-K_{u \Phi}{ }^{T} U\right]
$$

Then, substituting Eq. 5 into Eq. 4, the following relation is obtained,

$$
\begin{aligned}
& M \ddot{U}+K_{u u} U=-K_{u \Phi} K_{\Phi \Phi}{ }^{-1} G+K_{u \Phi} K_{\Phi \Phi}{ }^{-1} K_{u \Phi}{ }^{T} U \\
& M \ddot{U}+\left[K_{u u}-K_{u \Phi} K_{\Phi \Phi}{ }^{-1} K_{u \Phi}{ }^{T}\right] U=-K_{u \Phi} K_{\Phi \Phi}{ }^{-1} G
\end{aligned}
$$

which, can be written as

$$
M \ddot{U}+K U=T_{G \Phi} G
$$

where

$$
\begin{gathered}
K=K_{u u}-K_{u \Phi} K_{\Phi \Phi}^{-1} K_{u \Phi}^{T} \\
T_{G \Phi}=-K_{u \Phi} K_{\Phi \Phi}{ }^{-1}
\end{gathered}
$$

Eq. 8 shows the relation between the applied charge $(\mathrm{G})$ to the piezo actuators and the resulting displacement $(\mathrm{U})$ in the glass plate. The difference in electric charge creates a potential difference in the piezo actuators, which causes the actuator to bend and the glass plate to deform. If the charge is applied to the piezo patches in the form of a sinusoidal signal, $G=|G| e^{j \omega t}$, the resulting displacements in the glass plate will be also a sinusoidal signal with a phase delay of $\Psi, U=|U| e^{j \omega t+\Psi}$. Then, we can obtain the frequency response function (FRF) as $H(\omega)=U(\omega) / G(\omega)$.

\section{$4 \quad$ Results}

Modal analysis was performed on the glass plate for 3 different boundary conditions; a) the glass was fully clamped from all edges, b) the long edges of the plate were 


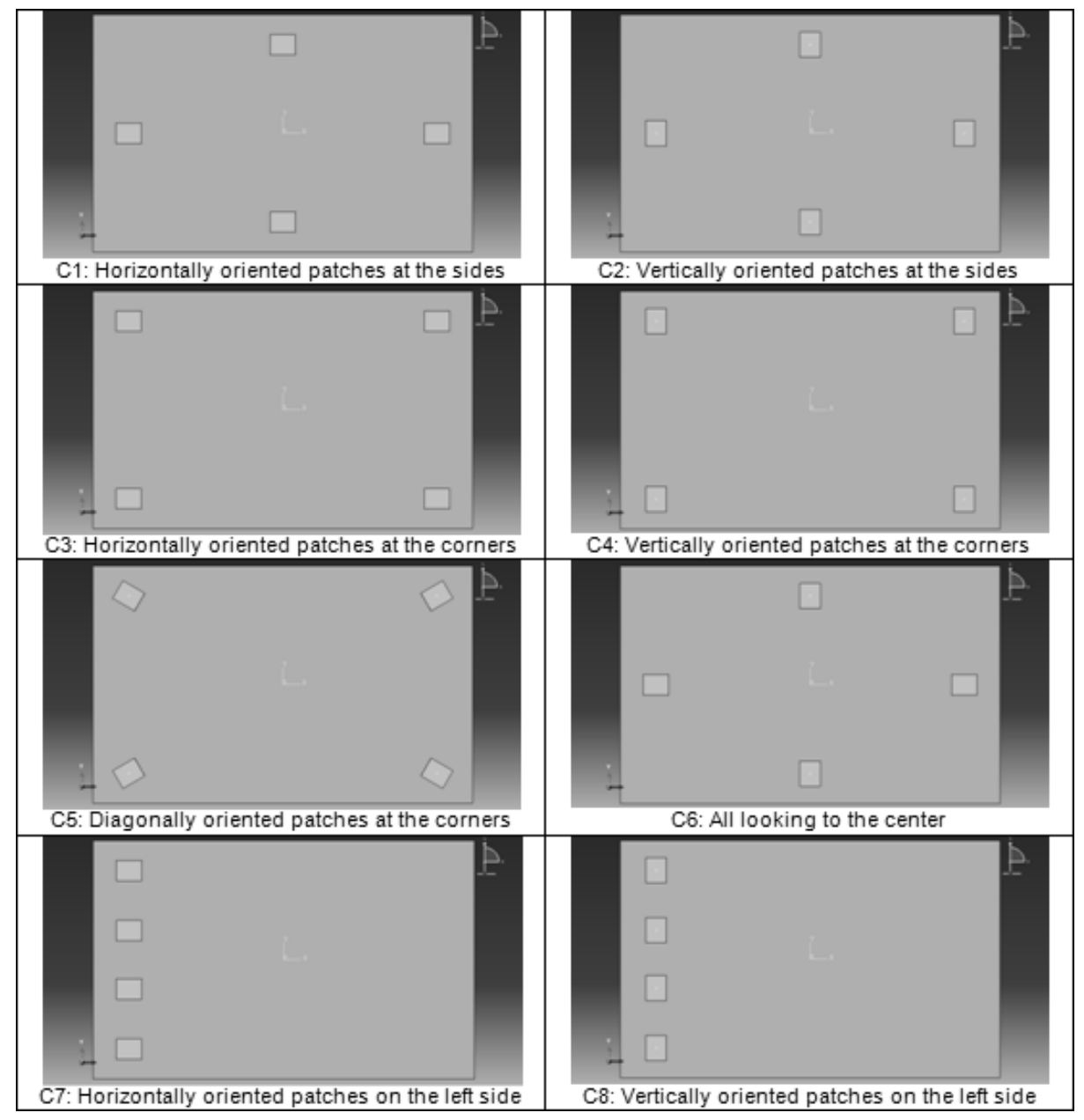

Fig. 3. The selected locations and orientations of the piezoelectric patches for the FE analysis

clamped while the short edges were left free, and c) the short edges of the plate were clamped while the long edges were left free. The first four mode shapes of the glass plate are also shown in Figure 4. The results obtained from ABAQUS are compared to the theoretical values [12] in Table 1 . Since we aim to vibrate the glass plate at frequencies that are within the limits of human vibrotactile perception of $0.1-500 \mathrm{~Hz}$ [13], the third boundary condition was chosen for the further analysis.

Then, the out of plane displacements of the glass plate were calculated at 15 different locations on the surface of glass plate (Figure 5), covering an area of $153 \mathrm{~mm}$ by $77 \mathrm{~mm}$. The frequency response function (FRF) of each point labeled as 1 to 15 in Figure 5 was calculated for the 8 different piezo configurations. As an exemplar, we show the FRFs of point 8 (center point) in Figure 6 for the $\mathrm{x}, \mathrm{y}, \mathrm{z}$ axes. 
Table 1. The first four resonance frequencies of the glass plate for the 3 different boundary conditions

\begin{tabular}{|c|c|c|c|c|}
\hline $\begin{array}{l}\text { Boundary } \\
\text { Conditions }\end{array}$ & $\begin{array}{c}\text { First } \\
\text { Resonance } \\
(\mathrm{Hz})\end{array}$ & $\begin{array}{c}\text { Second } \\
\text { Resonance } \\
(\mathrm{Hz})\end{array}$ & $\begin{array}{c}\text { Third } \\
\text { Resonance } \\
(\mathrm{Hz})\end{array}$ & $\begin{array}{c}\text { Fourth } \\
\text { Resonance } \\
(\mathrm{Hz})\end{array}$ \\
\hline a) & $\begin{array}{c}\text { ABAQUS: } \\
690.29 \\
\text { Theoretical: } \\
706.67\end{array}$ & $\begin{array}{c}\text { ABAQUS: } \\
1194.00 \\
\text { Theoretical: } \\
1204.00\end{array}$ & $\begin{array}{c}\text { ABAQUS: } \\
1605.30 \\
\text { Theoretical: } \\
1637.50\end{array}$ & $\begin{array}{c}\text { ABAQUS: } \\
2022.40 \\
\text { Theoretical: } \\
1865.10\end{array}$ \\
\hline b) & $\begin{array}{c}\text { ABAQUS: } \\
523.02 \\
\\
\text { Theoretical: } \\
526.13\end{array}$ & $\begin{array}{c}\text { ABAQUS: } \\
588.56 \\
\text { Theoretical: } \\
591.87\end{array}$ & $\begin{array}{c}\text { ABAQUS: } \\
830.60 \\
\text { Theoretical: } \\
846.17\end{array}$ & $\begin{array}{c}\text { ABAQUS: } \\
1326.50 \\
\text { Theoretical: } \\
1225.00\end{array}$ \\
\hline c) & $\begin{array}{c}\text { ABAQUS: } \\
318.46 \\
\text { Theoretical: } \\
321.38\end{array}$ & $\begin{array}{c}\text { ABAQUS: } \\
417.19 \\
\text { Theoretical: } \\
418.52\end{array}$ & $\begin{array}{c}\text { ABAQUS: } \\
824.88 \\
\text { Theoretical: } \\
772.66\end{array}$ & $\begin{array}{c}\text { ABAQUS: } \\
881.28 \\
\text { Theoretical: } \\
964.60\end{array}$ \\
\hline
\end{tabular}

\section{Discussion}

The goal of our project is to produce controlled vibrations on the surface of a touch screen via piezo actuators attached to its surface. To achieve higher vibration amplitudes, higher voltages must be applied to the actuators, which results in higher energy consumption. However, most of the devices utilizing interactive touch screens, especially the mobile ones, are limited by power. Currently, there are no established methods on a) how many piezo patches must be used and b) how they must be attached to a touch screen to generate the desired haptic effects with minimum power. Since the piezo patches cannot be easily detached from a touch screen once they are glued to its surface, it is more convenient to make this analysis in simulation environment using a finite element package. In fact, this is the approach followed in this paper.

As shown in Figure 7, the displacement amplitudes of the center point (point 8 in Figure 5) at each resonance frequency is different for the 8 different piezo configurations. To further investigate the effect of the piezo configurations on the vibration amplitude of each measurement point for a unit micro Coulomb charge applied to 


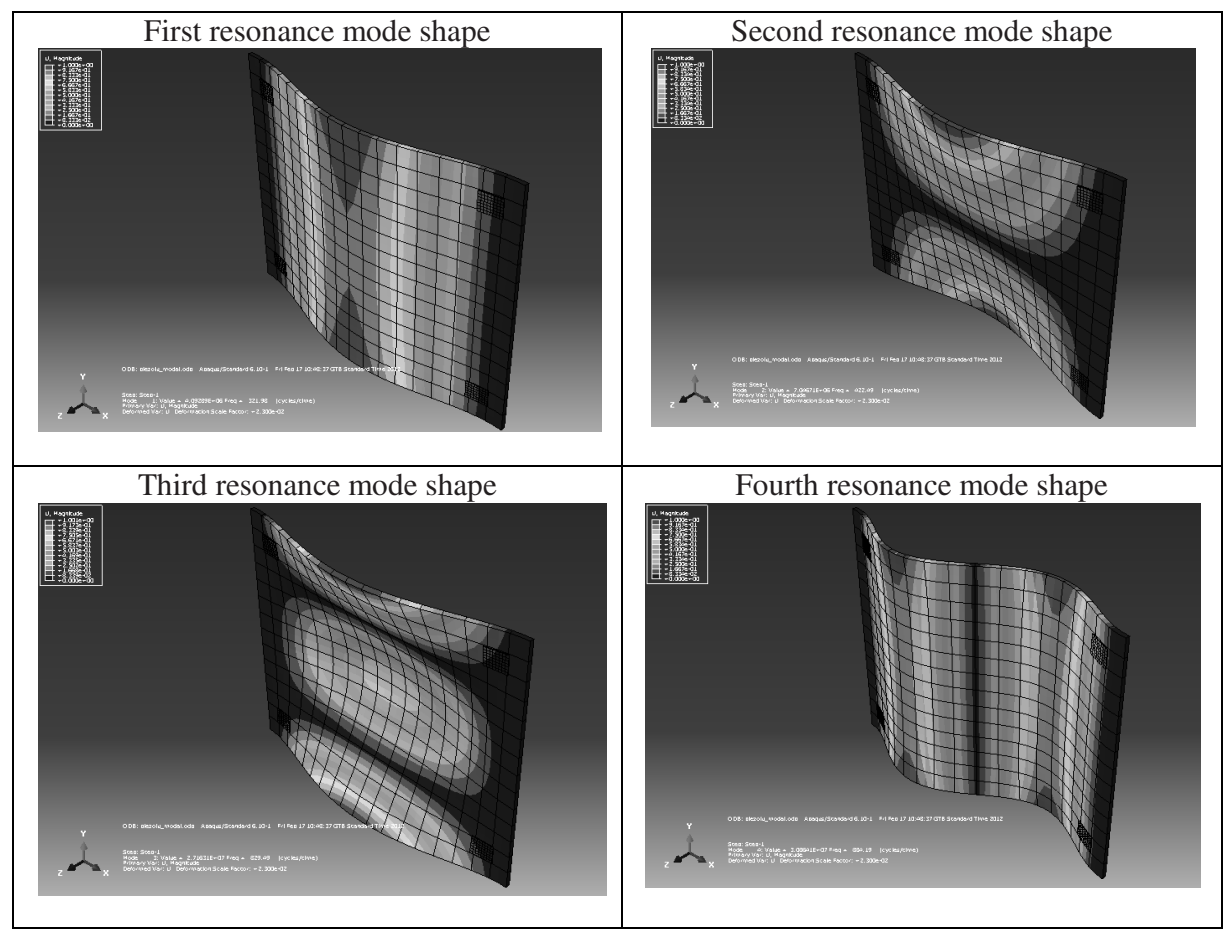

Fig. 4. The first four mode shapes of the glass plate

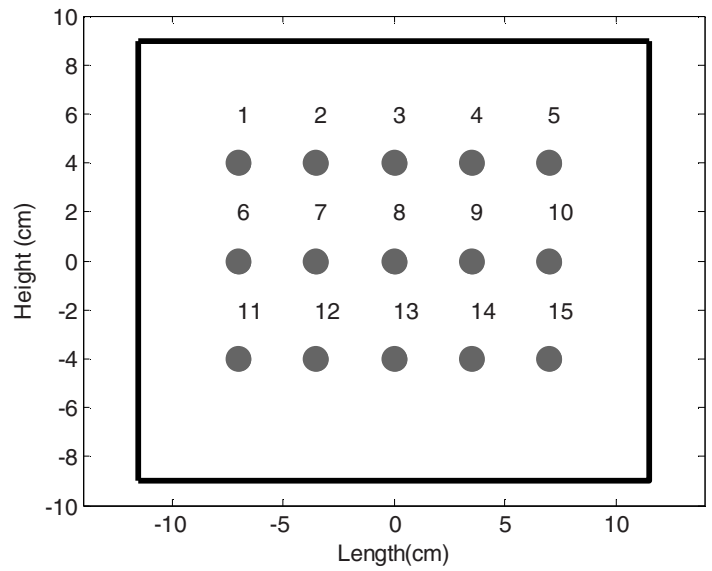

Fig. 5. Selected measurement points on the glass plate for the FE analysis 




Fig. 6. The frequency response function (FRF) of the point 8 (the center point on the glass plate) for $\mathrm{x}, \mathrm{y}$ and $\mathrm{z}$ axes. The first four resonance frequencies of the plate are marked on the figure.

each piezo (approximately equivalent to $100 \mathrm{~V}$ ), the plot shown in Figure 8 was constructed from the FRFs. It is obvious from Figure 8 that some of the piezo configurations (C3, C4, C5, C7 and C8) lead to more displacement at the measurement points than the others. If the locations of the patches are inspected carefully for these configurations (see Figure 3), one can conclude it is preferable to place the patches close to the fixed boundaries of the glass plate and not close to its free boundaries. In fact, if the third and fourth resonances of the plate are also considered for the analysis (Figure 7), the piezo configurations $\mathrm{C} 3, \mathrm{C} 4$, and $\mathrm{C} 5$ are even more favorable.



Fig. 7. The out-of-plane (z-axis) displacement amplitudes of the point 8 (the center point on the glass plate) for the first 4 resonance frequencies 


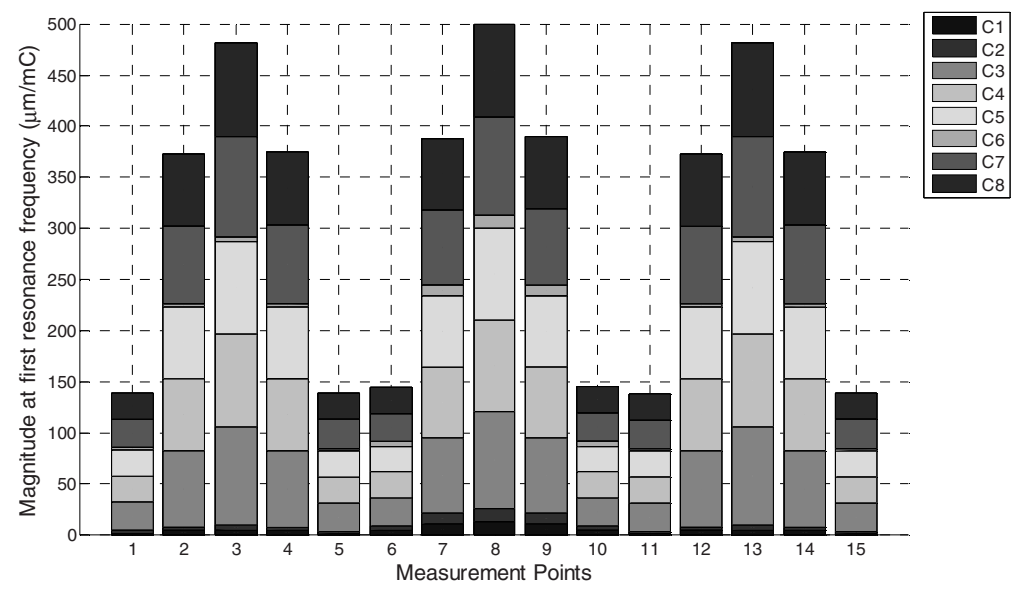

Fig. 8. The bar chart shows the vibration amplitude of each measurement point at the first resonance frequency for the 8 different piezo configurations

\section{Future Work}

To validate the results obtained through the finite element model, experimental modal analysis will be performed with four piezo patches attached to the glass plate. Then, user studies will be conducted to investigate the human haptic perception of various textures displayed on the glass plate by altering the frequency and the amplitude of the vibrations.

Acknowledgement. This work was supported by Turk Telekom under Grant Number 11315-01.

\section{References}

1. Kaczmarek, K.A., Tyler, M.E., Bach-y-Rita, P.: Electrotactile Haptic Display on the Fingertips: Preliminary Results. In: Engineering in Medicine and Biology Society, Engineering Advances: New Opportunities for Biomedical Engineers, Proceedings of the 16th Annual International Conference of the IEEE, vol. 2, pp. 940-941. IEEE (1994)

2. Kaczmarek, K.A., Nammi, K.K., Agarwal, A.K., Tyler, M.E., Haase, S.J., Beebe, D.J.: Polarity Effect in Electro-vibration for Tactile Display. IEEE Transactions on Biomedical Engineering 53(10), 2047-2054 (2006)

3. Yamamoto, A., Nagasawa, S., Yamamoto, H., Higuchi, T.: Electrostatic Tactile Display with Thin Film Slider and Its Application to Tactile Telepresentation Systems. IEEE Transactions on Visualization and Computer Graphics 12(2), 168-177 (2006)

4. Bau, O., Poupyrev, I., Israr, A., Harrison, C.: TeslaTouch: Electrovibration for Touch Surfaces. In: 23rd ACM Symposium on User Interface Software and Technology, pp. 283-292. ACM (2010) 
5. Fukumoto, M., Sugimura, T.: Active Click: Tactile Feedback for Touch Panels. In: Conference on Human Factors in Computing Systems, pp. 121-122. ACM, New York (2001)

6. Poupyrev, I., Maruyama, S.: Tactile Interfaces for Small Touch Screens. In: 16th ACM Symposium on User Interface Software and Technology 2, pp. 217-220. ACM, New York (2003)

7. Biet, M., Casiez, G., Giraud, F., Lemaire-Semail, B.: Discrimination of Virtual Square Gratings by Dynamic Touch on Friction Based Tactile Displays. In: Symposium on Haptic Interfaces for Virtual Environment and Teleoperator Systems, pp. 41-48 (2008)

8. Winfield, L., Glassmire, J., Colgate, J.E., Peshkin, M.: T-PaD: Tactile Pattern Display through Variable Friction Reduction. In: 2nd Joint EuroHaptics Conference and Symposium on Haptic Interfaces for Virtual Environment and Teleoperator Systems, pp. 421-426. IEEE (2007)

9. Chubb, E.C., Colgate, J.E., Peshkin, M.A.: ShiverPad: A Device Capable of Controlling Shear Force on a Bare Finger. In: 3rd Joint EuroHaptics Conference and Symposium on Haptic Interfaces for Virtual Environment and Teleoperator Systems, pp. 18-23 (2009)

10. Piefort, V.: Finite Element Modeling of Piezoelectric Active Structures. Ph.D. thesis, Faculty of Applied Sciences, Universit'e Libre de Bruxelles, Belgium, p. 87 (2001)

11. IEEE Standard on Piezoelectricity. ANSI/IEEE Std. 176-1987. IEEE (1988)

12. Blevins, R.D.: Formulas for Natural Frequency and Mode Shape. Krieger, Florida (1984)

13. Jones, L.A., Sarter, N.B.: Tactile Displays: Guidance for Their Design and Application. Human Factors: The Journal of the Human Factors and Ergonomics Society 50, 90-111 (2008) 